\title{
Mobile Learning Applications: Exploring Location Sensing Mechanisms
}

\author{
Cecilia Challiol $^{1,2[0000-0001-5140-0264]}$, Alejandra B. Lliteras ${ }^{1,3[0000-0002-4148-1299]}$ and Silvia \\ E. Gordillo ${ }^{1,3[0000-0001-5724-5058]}$ \\ ${ }^{1}$ UNLP, Facultad de Informática, LIFIA. 50 y 120 s/n, La Plata, Buenos Aires, Argentina \\ ${ }^{2}$ CONICET. Argentina \\ ${ }^{3}$ CICPBA. Buenos Aires, Argentina \\ \{ceciliac,lliteras,gordillo\}@lifia.info.unlp.edu.ar
}

\begin{abstract}
In this paper, we present an exploration of two location sensing mechanisms, such as QR codes and beacons, used in Location-based Learning Applications which are performed inside small indoor spaces (e.g. a classroom). The exploration of QR codes was carried out through an implementation of a Location-based Learning Application which was used by students between 7 and 11 years old inside of Primary Schools. Meanwhile, for the exploration of beacons, a functional prototype was made which was systematically tested by students of Faculty of Informatics of UNLP (Argentina). We analysed those characteristics that we have been learned related to how these mechanisms behave when they are used inside small indoor spaces because, in these cases, relevant places to provide learning contents are nearest from each other. We wish to create a discussion about the exploration of these two location sensing mechanisms. We expect that this discussion helps not only to design and develop Location-based Learning Applications, but also to improve authoring tools that are using to generate this kind of applications.
\end{abstract}

Keywords: Location-based Learning Applications, Sensing Mechanisms, QR codes, Beacons, Learning Content, Mobile Learning, HCI.

\section{Introduction}

Mobile Applications have been growing over the last years due to technological improvement [1]. Nowadays, there are different kinds of mobile applications, those that consider user's location to provide information or services according to his/her location are called Location-based Applications. In [2], this kind of applications is classified as: tour guides, learning tools, location-aware fiction and location-aware games. However, fictions or games also could be combined with learning (or educational) applications or tools. Different patterns to structure the content of these applications are described in [2]. Thus, each pattern provides to user different kind of interaction.

In Location-based Applications are defined relevant places (of a physical space) where users receive some kind of interaction [3]. In particular, Location-based Learning Applications provide, in these relevant places, learning content to students 
through their mobile devices. For example, a learning content could be a question [4]. Related to this kind of applications is involved location sensing mechanisms which allow to sense student's location. So, when a sensed location matches to a learning content, this content is provided to students.

Sensing mechanisms could be classified, for example, as direct or indirect [5], depending on whether they demand some sort of explicit users interaction. Direct mechanisms do not require any users interaction, for example GPS. However, indirect mechanisms require users interact in some way, as for example, QR codes [6] which need users read explicitly the code with their mobile devices. On the other hand, according to [1], contextual data (which include user's location) could be collected in different ways: physical sensors (e.g. GPS and QR code reader), digital sensors (as the calendar of mobile devices), social networks or be input manually by users. Note that, some of these ways of collect contextual data are direct while others are indirect (requiring some user interaction).

The goal of this paper is to present an exploration of two location sensing mechanisms, such as QR codes and beacons [7] (which make used of Bluetooth Low Energy -BLE- technology). These mechanisms in this exploration are used in Location-based Learning Applications which are performed inside small indoor spaces (e.g. a classroom). So, learning activities are carried out completely inside of a classroom. This means that relevant places to provide learning contents are nearest from each other. Note that, these activities are provided to students through Location-based Learning Applications. In this paper is analysed those characteristics that we have been learned related to how these mechanisms behave when they are used inside small indoor spaces. We wish to create a discussion about the exploration of these two location sensing mechanisms. We expects that this discussion helps not only to design and develop Location-based Learning Applications, but also to improve authoring tools that are using to generate this kind of applications.

The exploration of QR codes (indirect sensing mechanism) was carried out through an implementation of a Location-based Learning Application which was used by students between 7 and 11 years old inside of Primary Schools. We have considered that all learning tasks of this application are referred to curricular contents previously presented to students by their teachers. Meanwhile for the exploration of beacons (direct sensing mechanism), a functional prototype was made as part of "Locationbased Learning Activities" project (in the context of "Projects of Development and Innovation Applications with Students 2017" of the Faculty of Informatics of UNLP, Argentina). This functional prototype was systematically tested by students who were participating of this project. In particular, this paper presents how these two sensing mechanisms behave when they are used inside a small indoor space, such as a classroom.

The paper is structured as follows. In Section 2, we describe some related works. In Section 3 is presented our exploration of two location sensing mechanisms when they are used inside small indoor spaces. Some discussion issues are described in Section 4. Conclusions and some future works are presented in Section 5. 


\section{Related Works}

In this section some related works to the topic of this paper are presented. To help the reader, some specific characteristics of how location sensing mechanisms work are detailed below, especially when they are used from mobile applications. These mechanisms can be classified or grouped in different ways. According to [5], one way of grouping is if these are direct or indirect mechanisms. This kind of classification is related to interaction involved in each mechanism. Direct mechanisms do not require any users interaction, for example GPS. When a mobile application uses this kind of direct sensing mechanism to detect the user's location, it is "listening" for new sensing values; whenever a new value is detected, updated information is automatically provided to users (according to their new location). This kind of applications are known as context-aware mobile applications [2] which have to implement the behavior to react automatically to changes received by each sensing mechanism.

Indirect sensing mechanisms require users interaction in some way, as it is the case of QR codes [6] in which users read codes explicitly with their mobile devices. Mobile applications that use this kind of indirect sensing mechanism (to detect user's location) provide to users some way to interact with the application, in order to detect a new sensing value. For example, if QR codes are used to sense user's location, the mobile application should provide a way to access to a QR code reader (which should be previously installed on the mobile device). When a user reads a new code from this mobile application, a QR code reader opens; then the reader detects a new code, so, the application reacts by providing information or services. Thus, it can only be possible to detect a location changes when user reads a new QR code. Another example of indirect location sensing could be when users indicate where they are at each moment, for example, by choosing from a list of possible places. In this case, it can not be possible to detect location changes until user selects a new item from this list.

According to the description above, it can be notice how depending on the kind of sensing mechanism users receive different form of interaction from mobile applications. In direct mechanisms, there are not interventions required from users to sense their current location. This is one of the reasons why they are often more used due to facilitate how to users interact with the application. Below some location sensing mechanisms used in the area of learning are presented.

GPS massification has allowed emerging Location-based Learning Applications for outdoor spaces, for example, in the domain of natural sciences, some applications of this kind are described in [8]. In [9], augmented reality application is analysed in which locations are determined by printed symbols and natural markers. The authors mention some problems of these mechanisms, for example, how sunlight affects to detect natural markers. Another augmented reality application is presented in [10], but in this case inside of a museum where locations are determined by beacons. These beacons are strategically located in relevant places of the museum with considerable separation from each other to avoid signal overlapping. When this application detects that user is near to a one of these beacon, he/she receives augmented reality information related to that place. On the other hand, in [11] are shown Location-based Learning Applications for indoor and outdoor spaces. These applications use Google 
Map for outdoor spaces [12] and GPS as a location sensing mechanism, while for indoor spaces are used static images and locations (for these spaces) are indicated manually by the students. That is to say, in [11] for the indoor spaces are using an indirect sensing mechanism; until students do not indicate where they are, the information related to their current location is not updated. So, the description mentioned above indicates Location-based Learning Applications could be use different types of sensing mechanisms to determine student's location, some direct and others indirect [5]. In fact, these applications could be evolve and change their sensing mechanisms over time. So, it depends on how they were initially designed, this evolution will have a minimal impact as mentioned in [13].

Teachers' participation [14] is fundamental for the design and creation of Locationbased Learning Applications. According to this, in recent years has begun to be explored different approaches or tools to facilitate teachers' participation in this task. In [4], four phases related to Location-based Learning Applications are identified: conceptual design, development cycle, put into practice the development and put into practice's evaluation. For the conceptual design phase, in [4], a conceptual framework is proposed for co-design this kind of applications which is focused particularly on the reuse of both learning contents and relevant locations. This conceptual framework uses the vision of separation of concerns (used in Software Engineering) to address co-design, in which at least teachers and technology experts are involved. On the other hand, in [11] is proposed to design these applications using the puzzle metaphor. The pieces are built, and then there are converted to XML files [15] to generate functional applications. In [11] is describe how this metaphor is used and shown how to use the generated applications. The authors mention in [11] that metaphors, in general, are widely used in the area of Human-Computer Interaction (HCI) to be able to handle unknown contexts from known concepts. For this reason, the authors use puzzle metaphor.

Achieving teachers have total control to design and create this kind of applications requires having authoring tools (for example, applications or Web sites) which help them to integrate location sensing mechanisms [16] without demand technical knowledge thereof. That is to say, it would be desirable that these kind of authoring tools allow teachers to create a mobile learning application that uses, for example, GPS without needing to know technical details of it. In this case, authoring tools should handle all technical and packaging details to generate mobile applications. Thus, teachers should only focus on creating and defining relevant locations where they want students receive learning content. Then, this is taken by the authoring tool to generate mobile learning applications which students use later on their mobile devices.

In order to create authoring tools, in particular for Location-based Learning Applications, an exploration of sensing mechanisms is required to provide a solution that does not demand teachers to know technical details of these. In recent years different authoring tools have been emerging which give a high level of abstraction of technical details for different location sensing mechanisms. For example, in [16] an authoring tool is described which contains a catalogue of beacons. This make easier, for teachers, to associate learning content with them. Previously, beacons have been strategi- 
cally placed in the physical space in order to avoid signals interfere with each other. Then, experts in technologies have measured all beacons' signal to achieve that the tool considers this information to facilitate teachers how to use them. So, authoring tool shows available beacons and teachers choose one of them to associate learning content. In this way learning contents are located. For this, teachers do not require to know any technical detail of the beacons. On the other hand, in [3] is presented SituAR which is a platform to create augmented reality content. Users are creators of their own stories and they do not require programming knowledge to generate them. This platform use GPS to associate location to each content. This association is not visible for the users. Every time user creates content, this is associated automatically with the current GPS location. Another authoring tool is SmartZoos [17] which allows designing content as modular open educational resource. Then, these resources are combined with location (latitude-longitude) which is obtained using a map. For this, SmartZoos provides Google maps which allow users to select relevant places in the physical space and associate them with content. This is used by SmartZoos to generate applications which make use of GPS as location sensing mechanism. On the other hand, in [18] an in-situ authoring tool for indoor-outdoor Location-based Learning Applications is presented. This tool defines learning contents and locations in a decoupled way, according to [4]. This information (learning contents and locations) are exported to XML files [15]. These files could be used to generate functional applications. The sensing mechanisms used by the tool defined in [18] are GPS and QR codes.

According to previous description about authoring tool, it could be appreciated that each of them focuses on some kind of sensing mechanisms. This is because of complexity of embedded sensing mechanisms (as part of tool) in a way that they do not require any type of technical expertise from users. To do that, these mechanisms should be explored enough, in order to understand how to use them in a simple way for any user. We hope that the exploration of sensing mechanisms presented in this paper would help to enhance authoring tools for this kind of applications.

\section{$3 \quad$ Exploring two sensing mechanisms in Location-based Learning Activities inside small indoor spaces}

In this section, we present an exploration of two location sensing mechanisms, such as QR codes and beacons. In particular, these mechanisms have been tested inside a small indoor space such as a classroom. The Learning Activity defined in [19] has been used for the exploration presented in this paper. This activity is composed of four tasks (three of collection and one of deposit) which have been designed for students between 7 and 11 years. Knowledge required to resolve these tasks are previously presented to students by their teachers because this knowledge is part of the curricular content of the course. So, one way to put this knowledge into practice was through these tasks. Each task presents some question to students. For collection tasks, students should pick concrete elements up that can be considered in response to a received question. Deposit tasks imply to put these collected elements on a contain- 
er according to predominant type material with that each of them has been produced (e.g. paper, metal or plastic). In [19], this Learning Activity is defined to encourage reuse not only each task but also each location. In Fig. 1 is shown an example of collection task, its question, and its elements. Additionally, this task can be associated, in another layer, with a particular location of the physical space.

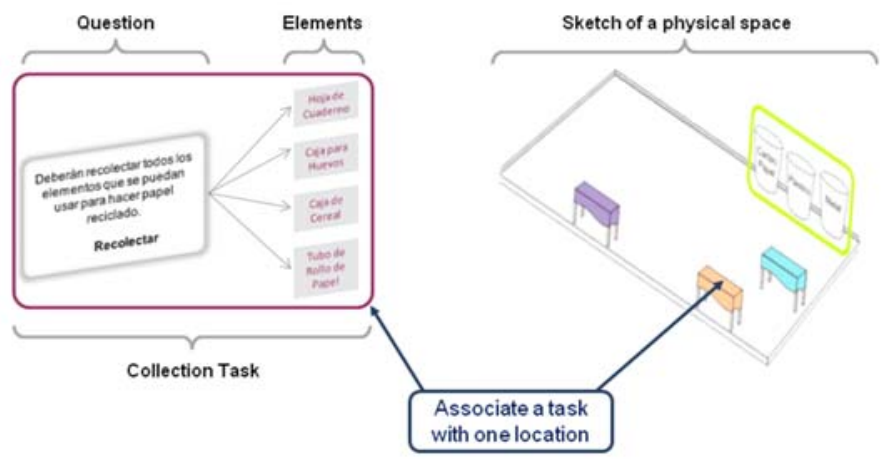

Fig. 1. A collection task associated with one location.

In the physical space visualized in Fig. 1, there are three relevant places related to collection tasks (tables). It also is shown a fourth relevant place related to a deposit task (containers). These places (locations) are defined independently of tasks which can be offered there. This allows reusing these places.

It should be noted that the Learning Activity presented in [19] is defined independently of the technological platform. So, it can be implemented using any sensing mechanism to determine student's location. That is, relevant places or locations are defined generically [13] in order to be combined with any location sensing mechanism. This allows that the same Learning Activity can be implemented with different sensing mechanisms. More details of how this Learning Activity was designed could be read in [19].

The Learning Activity mentioned above has been used as a base to implement a Location-based Learning Application and a functional prototype. The first one uses QR codes to sense the student's location while the second one uses beacons to do that.

Exploration performed related to these sensing mechanisms (QR codes and beacons) is described below. It should be noted that, in both cases, all relevant places (where questions of each task are provided) are inside a small indoor space. In particular, they are in the same classroom as is shown in Fig. 1.

\subsection{Exploration of the use of $Q R$ codes in the implementation of a Location- based Learning Application}

The exploration of QR codes (indirect sensing mechanism) was carried out through an implementation of a Location-based Learning Application which was used by students between 7 and 11 years old inside of Primary Schools. This application is called 
"Aprendo Jugando". It should be noted that this application was implemented as a proof of concepts for validate a modeling approach proposed in [19]; this approach is for model location-based learning activities which consider concrete elements. Owing to "Aprendo Jugando" features, physical space requires to be configured previously because tables (with their elements) and containers need to be located in specific places before students start to use the application. This is shown in Fig. 2. Note that, tables have their concrete elements to be collected in each task.
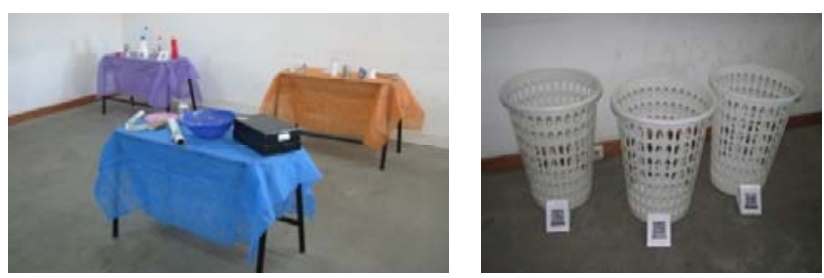

Fig. 2. Physical space configuration for "Aprendo Jugando".

"Aprendo Jugando" was developed for Android version 4.0 and uses QR codes. On the one hand, these codes are used (for a group of students) to receive the question of a task (to collect or deposit). On the other hand, these codes are used (by this group) to indicate what concrete element is picked up in response to a collect task. That is, a group of students should read a QR code to receive a question and after that, they should read QR codes of concrete elements (see Fig. 3) to collect them. Collecting elements in this way produces that each of them is put in a virtual basket of the application. On the other side, a group should pick each concrete element (corresponding with the QR code read) up, and they put them in a physical basket. So, this means that virtual basket should reflect what a group has in their physical basket.

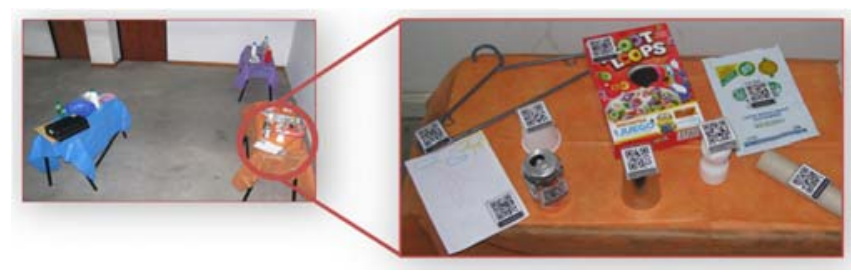

Fig. 3. Concrete elements of a collect task for "Aprendo Jugando".

Suppose, a group decide that a particular concrete element that was collected is not correct in order to respond to the question of their current task. If the group decides to return the collected element, then they should select this element from a list displayed on their mobile device, so, this indicates that they want to remove it from the virtual basket of collected elements. Then, they should touch the delete option. In this way, this element is removed from the virtual basket. In addition, the group has to find this concrete element inside their physical basket, and return it to their current task's table. 
It should be noted that to perform a collection task, as mentioned above, a group should read the QR code of the element if they decide to collect it. However, to deposit an element into a container, a group should select an element from a list displayed on their mobile device, and also, they should pick this concrete element up from their physical basket. Then, they deposit it into a container which they think is appropriate. That is, deposit task does not involve to read the element's QR code. So, this works in a similar way to return an element because a group notes that this does not respond to their current task (as it is explained above).

According to the description above, it is noted that concrete elements were on the tables are moved (or transported) to some of the containers. Thus, every time a group finishes to use "Aprendo Jugando", physical space should be reconfigured as shown in Fig. 2.

In [19], authors describe how they have putted into practices of "Aprendo Jugan$d o$ " in two primary schools. In this paper, these putting into practices will be analysed from the QR codes use perspective. It should be noted that each putting into practice was carried out with groups of three or four students. Each group have been formed freely among themselves and they had a single mobile device. The latter mentioned allows taking advantages of "face to face" collaboration to solve tasks [20]. This also allows establishing social interactions and thus favouring knowledge circulation for collaborative building of it [21].

During first putting into practice it was detected that first time that a group had to read a QR code, this was a complex action. This situation is due to the fact that use QR codes require a level of indirection to obtain a learning content. Reading a QR code involves a conscious act of students once QR codes reader application (which is embedded in the "Aprendo Jugando") is opened. Then, students should focus the mobile device camera, so, that it "reads" a QR code. This could be appreciated in Fig. 4, where a student reads a QR code of a task and then he/she receives information of the question associated with it (on the screen of mobile device). In this received screen, a group has a collecting elements option, when they select this option, a QR code reader is opened which allows to read QR codes of concrete elements that the group want to collect in response to their current task.

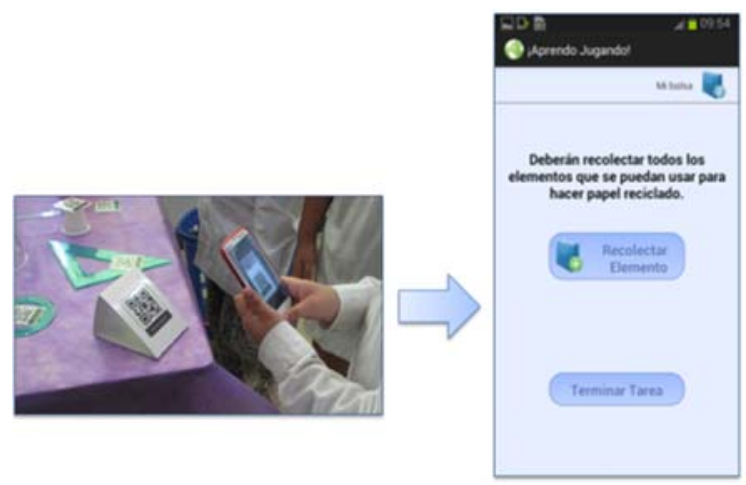

Fig. 4. Student reading a QR code of a collection task. 
After second or third times that a groups interacted with this way of reading QR codes, they incorporate it without problems, because they understand how this work. In spite of, there are two different semantics associated to when QR code reader is used, one to receive the question and another to collect concrete elements, this did not impact to students when they have been using the application. The difficulty was only detected to understand how QR codes interaction was, in general, the first time.

In the first putting into practice was detected the lack of naturalness of reading $Q R$ codes for groups which as mention before involving indirect interaction with the QR code reader. For this reason, we have decided to add a scaffolding activity for the second putting into practice in a school. For this, a reduced micro application was developed only to show how to read QR codes. This micro application is called "Exploro como Jugar" which have been used in a students group talk in the second school before each group uses the "Aprendo Jugando". This small variation (from first to second putting into practice) made it easier for students to read QR codes for the first time in "Aprendo Jugando" without any problem. So, students experience to read QR codes have been improved.

In this way, it can be seen that in the case of using an indirect sensing mechanism and that this requires an unknown form of interaction for students, it would be advisable to first explain it. This would mean that during the putting into practice of the application (in this case, "Aprendo Jugando") the form of interaction does not become a limiting factor, thus avoiding frustrations and demotivation on the part of the students. This explanation would not be viable when what one wants to prove is either the naturalness of the interaction of the sensing mechanism for the students, where in this particular case, a previous training could affect the results.

\subsection{Exploring the behaviour of the beacons in a functional prototype}

After carrying out the putting into practices of "Aprendo Jugando" application [19], it was possible to register QR codes interaction experience. This interaction requires that a user "read" a code with their mobile devices which "can be a tedious process to learn and apply" [22] or "unintuitive" [10]. In [10] and [22], authors propose to use beacons instead of QR codes. In [7] is described the use of beacons as natural evolution of QR codes.

Considering the description mentioned above, we had decided to develop a functional prototype which uses beacons only to receive each task. This have allowed to test a direct sensing mechanism to detect students' location avoiding students having to read a QR code to receive a question. In this way, when students approach to each table shown in Fig. 2, they automatically receive the corresponding question on their mobile device.

This functional prototype was systematically tested by students who were participants in "Location-based Learning Activities" project (in the context of "Projects of Development and Innovation Applications with Students 2017" of the Faculty of Informatics of UNLP, Argentina). 
These tests focused on analyze how to use this direct sensing mechanism and how beacons behaved when they were located on tables which are located inside small distances from each other.

In a small and limited indoor space as a classroom, beacons presented many anomalies. Detecting that depending on:

- Beacon's orientation, its signal is affected. For example, if a beacon is oriented horizontally (as shown Fig. 5.B), its signal decreases considerably respect to beacon's signal emitted when it is oriented vertically (see Fig. 5.A). This is because its signal is emitted from beacon's front. For this reason, beacons can often find placed on walls of places where they are used.

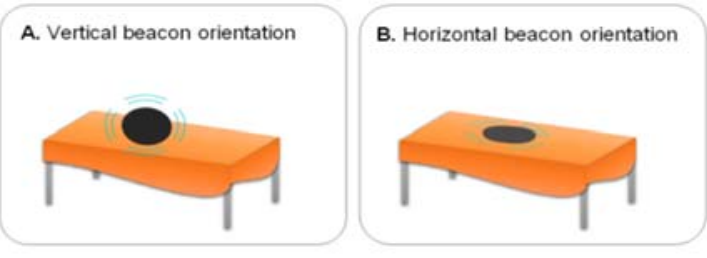

Fig. 5. Beacon's orientation on a table: Vertical or Horizontal.

- Mobile device's orientation, received beacon's signal could be not accuracy to the real distance to it. So, in this situation could do interpret user's location further away of real beacon's location. This is because mobile device's bluetooth is located on one side of it. So, if a beacon is located on the same side, its signal is received without problems. However, if a beacon is located on the other side, its signal decreases considerably, this makes interpret user's location further away of real beacons's location. For Location-based Learning Applications (used inside a small indoor space), this situation generates that many times an application interpret that students are in a table but in reality students are closer to another. In Fig. 6, it shown a mobile device locates at the same distance (one meter) from two beacons. However, when a Samsung Galaxy S4 device is used to test, the right beacon is closest. This is because its bluetooth sensor is on this side. This can vary by different mobile devices vendors and models. So, this behaviour affects how an application works, so, students could receive a question which is not appropriate to their nearest table because it was detected by another beacon due to mobile device's orientation. This kind of signal problem is addressed, for example, in [23] where authors advise to locate beacons to ten meters distance from each other. Thus, in this way they avoid signal conflict and it is easier to determine unambiguous locations. This condition, in case of a small indoor space, is not possible to accomplish. 


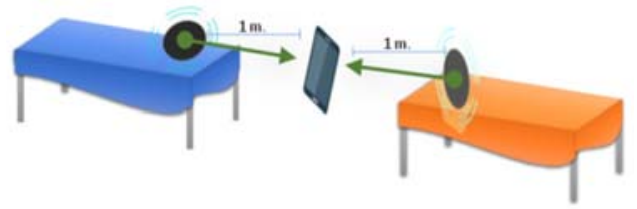

Fig. 6. Example of beacon located to right and left side of a mobile device.

- Processing capacity of mobile device, processing speed of beacons' signal could delay more to be analysed. This means depend on mobile device's vendor and model. So, user should be closer to a beacon to receive a corresponding learning task, since many times device is still processing a received signal while user is still walking. Our tests were conducted with a Samsung Galaxy S3 and a Samsung Galaxy S4. Detecting for first one device that beacons' signal is received later. So, if user kept moving he/she could receive a task when he/she was no longer near to the corresponding table.

Systematic tests carried out with this functional prototype has allowed to detect how it work in a small indoor space in which there are tables located at small distances from each other. For this mentioned case, this kind of direct sensing mechanism would not be the most recommended. Because, erroneous beacon's signal processing could trigger student receive a question which is not correspond with the real student's location. For example, student could be ask to collect certain concrete elements, but he/she is located another table (associated to another task), so, concrete elements over this table are not respond the question that he/she have received. Moreover, this kind of application's behavior could be imperceptible for students, since they may think that proposed question is suitable with the table closest to them. Take into account these results; we have decided not to advance beyond the functional prototype (which uses beacons). So, this prototype has not been put into practice with students in schools, as if we did with "Aprendo Jugando" which uses QR codes.

\section{Discussion}

In this section, some explored features related to location sensing mechanisms are discussed. Hoping that this discuss helps not only to design and create Location-based Learning Applications, but also to improve authoring tools using to generate this kind of applications.

When QR codes are using, it is indispensable to consider that code reader applications may have different sensitivity. This implies that by having a reading application open, any QR code could be read by it. This is designed in this way in order to speed up the user's task. However, when this mechanism is used inside applications, such as "Aprendo Jugando" where there are many concrete elements on a table, each of them with a QR code; so, one of them could be read without explicit intention. That is, it could be read a QR codes of a concrete element but may be this is not that student want. Because, they want not to collect this element. During putting into practices of 
"Aprendo Jugando", this situation mentioned before only happened to one of the groups. But, for this group this situation did not generate any inconveniences in the use of the application. It should be noted that QR code reader applications should be previously installed in a mobile device to be used in a mobile application, as it is the case of "Aprendo Jugando".

In the case of use beacons, it is advisable to locate them at a considerable distance from each other. Note that beacons' signal is interfered with by building characteristics (for example walls). So, for each physical space a technical test should be carried out in order to decide optimal distances according to these characteristics. If a catalog of beacons is provided, as [16], these should be placed strategically with the aim of avoid signal interfere from each other. This facilitates a proper functioning of applications based on them. In the case of beacons are inevitably require to be located close to each other, it could be possible to define an ad hoc heuristic to analyze which is the closest beacon in every moment. For this, it could use some variables such as device's orientation to evaluate if a user is on the left or on the right of the beacon. Sensing mechanisms based on signals usually function with sensed data's accuracy and a margin of error. These data allows to take better decisions (by applications) in order to decide what information or services be offer to the user. In addition, beacons emit values to indicate distance from mobile device to it; for example, one, two or three meters. So, in this case, it could decide to provide only learning content when a distance to a beacon is less than one meter. However, this last mentioned strategy to offer content not always work correctly since it depends on processing capacity of the mobile device. So, it could occur that a student is located less than one meter from a table but this situation is not detected by a mobile application which is still processing sensed data. Therefore, for each domain is required to analyze what could be the best ad hoc heuristic to process beacon's signals, and thus to determine which one is closest to a user all the time. However, it is very complex to achieve a generalized solution applicable to any physical space and any mobile device. Note that, for beacons to be detected by mobile devices, they should support BLE (Bluetooth Low Energy) technology.

Using QR codes to determine students' location affect application's visual interface. Because, a selectable option should be provided to open the corresponding reader (as shown in Fig. 4 to collect concrete elements). In the case of beacons, the use of them is transparent for students, since when they are near a beacon, they receive learning content (for example, a question) associated with this beacon. However, in direct sensing mechanisms, such as beacons, it should be consider the delay to receive associated information to them. Users are constantly walking, so, sometime they may receive outdated information to their current location, such as it is mentioned in [7].

It can be appreciated how each location sensing mechanism involves a different form of interaction and they require specific considerations according to how they behave. It is essential to perform in-situ functional tests to detect first if a location sensing mechanism is suitable for a physical space where a Location-based Learning Application is planned to use. Depending on this, it will be possible to perform then tests with students. 
It is interesting to note that, in the last recent years, authoring tools ([3], [16], [17] and [18]) to facilitate creation of applications which use sensing mechanisms has increased. Generating applications with these tools is very useful for teachers, but they also need to understand how sensing mechanisms work in order to choose the most appropriate for each situation. This may require teachers training in order to know how each location sensing mechanism behaves.

\section{Conclusions and Future Works}

An exploration of two location sensing mechanisms, such as QR codes and beacons, have been presented for Location-based Learning Applications inside small indoor spaces such as a classroom. It has been described how each mechanism behaves and some characteristics related to them have been discussed. For physical spaces where relevant locations are close to each other, beacons are not a recommended option to use as a location sensing mechanism. The exploration presented in this paper allows analyzing viability of each sensing mechanism when some specific requirements required to be covered, in this case to be used inside a small indoor space.

Taking into an account the four phases proposed [4] to the Location-based Learning Applications (conceptual design, development cycle, put into practice the development and evaluation of the put into practice), these four phases have been performed by the application presented in Section 3.A ("Aprendo Jugando"), which uses QR codes as location sensing mechanism. However, for the functional prototype presented in Section 3.B, which uses beacons as location sensing mechanism, have been carried out the two first phases. So, the third phase (put into practice the development) could not be performed with students in school because beacons are not suitable as location sensing mechanism for a small indoor space. According to this, it can be observed that the phases proposed in [4] could be carrying out only if it is viable to pass to the next phase. For example, if conceptual design is not feasible to develop (due to complexity or high cost involved in it), so, in this case, the rest of the phases defined in [4] could not be addressed either.

We are working on develop an API which allows to generalize sensing mechanisms behavior, in order to be easier to develop this kind of application. So, this allows that they can evolve over time without requiring too many changes. For this, we take into account what is proposed in [13] which describes a conceptual framework based on the separation of concerns and one of these concerns involve sensing mechanisms. In this way, applications could be "listening" for user's location sensing in general, so, it do not matter what mechanism is used to sense user's location.

In the future, we will be planned to organize meeting with multidisciplinary teams to register emerging features which could contribute to detect other requirements when this kind of applications are created. So, this will be use to provide tools that allow to speed up the creation of this applications. For example, expanding the features of the tool presented in [18]. These meetings are also expected to help understand how to carry out co-design of this kind of applications when a multidisciplinary team is involved. 


\section{Acknowledgments}

The authors thank Ramiro Ongaro, Juan Emilio Salaber and Andrés Gabriel Binaghi for their participation in systematic tests of the functional prototype in "Location-based Learning Activities" project (in the context of "Projects of Development and Innovation Applications with Students 2017" of the Faculty of Informatics of UNLP, Argentina), Res. HCD No 4/17, File No. 3300-006343/17-000).

\section{References}

1. Rivero-Rodriguez, A., Pileggi, P., Nykänen, O.A.: Mobile context-aware systems: technologies, resources and applications. International Journal of Interactive Mobile Technologies 10(2), 25-32 (2016). http://online-journals.org/index.php/i-jim/article/view/5367.

2. Hargood, C., Hunt, V., Weal, M.J., Millard, D.E.: Patterns of sculptural hypertext in location based narratives. In: 27th ACM Conference on Hypertext and Social Media (HT '16), pp. 61-70, ACM, New York, USA (2016). doi: 0.1145/2914586.2914595.

3. Vera, F., Sánchez, J.A.: A model for in-situ augmented reality content creation based on storytelling and gamification. In: 6th Mexican Conference on Human-Computer Interaction, pp. 39-42, ACM, New York, USA (2016). doi: 10.1145/2967175.2967385.

4. Lliteras, A.B., Challiol, C., Gordillo, S.E.: Location-based mobile learning applications: a conceptual framework for co-design. In: 12th Latin American Conference on Learning Technologies (LACLO), pp. 358-365, IEEE Press, La Plata, Argentina (2017), doi:10.1109/LACLO.2017.8120946.

5. Emmanouilidis, C., Koutsiamanis, R.A., Tasidou, A.: Mobile guides: Taxonomy of architectures, context awareness, technologies and applications. Journal of Network and Computer Applications 36(1), 103-125 (2013). doi:10.1016/j.jnca.2012.04.007.

6. Kato, H., Chai, D., Tan, K.T.: Barcodes for mobile devices. Cambridge University Press, New York, USA (2010). doi:10.1017/CBO9780511712241.

7. Hartmann, K., Quirnn, A.: How useful are BLE beacons for mobile guides?. In: 5th International Conference on Wireless Networks and Embedded Systems, pp. 1-10, IEEE Press, Rajpura, India (2016). doi: 10.1109/WECON.2016.7993487.

8. Zydney, J.M., Warner, Z.: Mobile apps for science learning: review of research. Computers \& Education 94, 1-17 (2016). doi: 10.1016/j.compedu.2015.11.001.

9. Alakärppä, I., Jaakkola, E., Väyrynen, J., Häkkilä, J.: Using nature elements in mobile AR for education with children. In: 19th International Conference on Human-Computer Interaction with Mobile Devices and Services, pp. 41-54, ACM, New York, USA (2017). doi:10.1145/3098279.3098547.

10. Tsai, T.H., Shen, C.Y., Lin, Z.S., Liu, H.R., Chiou, W.K.: Exploring Location-based augmented reality Experience in museums. In: Antona M., Stephanidis C. (eds) UAHCI 2017, LNCS, vol. 10278, pp. 199-209, Springer, Cham (2016). doi: 10.1007/978-3-319-587035_15.

11. Melero, J., Hernández-Leo, D.: Design and implementation of location-based learning games: four case studies with 'QuesTInSitu: The Game'. IEEE Transactions on Emerging Topics in Computing 5(1), 84-94 (2017). doi: 10.1109/TETC.2016.2615861.

12. Google Maps, https://maps.google.com, last accessed 2018/05/23.

13. Challiol, C., Lliteras, A.B., Gordillo, S.E.: Diseño de aplicaciones móviles basadas en posicionamiento: un framework conceptual. In: XXIII Congreso Argentino de Ciencias de la 
Computación (CACIC 2007), pp. 682-691, RedUNCI, La Plata, Argentina (2017). http://sedici.unlp.edu.ar/handle/10915/63780.

14. Cober, R., Tan, E., Slotta, J., So, H.J., Könings, K.D.: Teachers as participatory designers: Two case studies with technology-enhanced learning environments. Instructional Science 43(2), 203-228 (2015). doi:10.1007/s11251-014-9339-0.

15. XML, https://www.w3.org/standards/techs/xml\#w3c_all, last accessed 2018/05/23.

16. Hauge, J.B., Stefan, I.A., Stefan, A., Cazzaniga, M., Yanez, P., Skupinski, T., Mohier, F.: Exploring context-aware activities to enhance the learning experience. In: Dias J., Santos P., Veltkamp R. (eds) GALA 2017, LNCS, vol. 10653, pp. 238-247, Springer, Cham (2017). doi:10.1007/978-3-319-71940-5_22.

17. Pishtari, G., Väljataga, T., Tammets, P., Savitski, P., Rodríguez-Triana, M.J., Ley, T.: SmartZoos: modular open educational resources for location-based games. In: Lavoué É., Drachsler H., Verbert K., Broisin J., Pérez-Sanagustín M. (eds) EC-TEL 2017, LNCS, vol. 10474, pp. 513-516, Springer International Publishing (2017). doi:10.1007/978-3-31966610-5_52.

18. Zimbello, A.M., Alconada Verzini, F.M., Challiol, C., Lliteras A.B., Gordillo, S.E.: Authoring tool for Location-based learning experiences. In: 4th IEEE/ACM International Conference on Mobile Software Engineering and Systems, pp. 211-212, IEEE Press, Buenos Aires, Argentina (2017). doi: 10.1109/MOBILESoft.2017.32.

19. Lliteras, A.B.: Un enfoque de modelado de actividades educativas posicionadas que contemplan elementos concretos. Master Thesis, Faculty of Informatics, National University of La Plata, Argentina (2015). http://hdl.handle.net/10915/50030.

20. Christensne, B.C., Giakalis, A.P., Jørgensen, N.M., Poulsen, M.K., Rehm, M.: The effect of device number and role assignment on social group dynamics in location-based learning. In: 15th International Conference on Mobile and Ubiquitous Multimedia, pp. 297-305, ACM, New York, USA (2016). doi: 10.1145/3012709.3012711.

21. Suthers, D. D., Vatrapu, R., Medina, R., Joseph, S., Dwyer, N.: Beyond threaded discussion: Representational guidance in asynchronous collaborative learning environments. Computers \& Education, 50(4), 1103-1127 (2008). doi: 10.1016/j.compedu.2006.10.007

22. Ng, P.C., She, J. Park, S.: Notify-and-interact: a beacon-smartphone interaction for user engagement in galleries. In: IEEE International Conference on Multimedia and Expo, pp. 1069-1074, IEEE Press, Hong Kong, China (2017). doi:10.1109/ICME.2017.8019467.

23. Lin, X.Y., Ho, T.W., Fang, C.C., Yen, Z.S., Yang, B.J., Lai, F.: A mobile indoor positioning system based on iBeacon technology. In: 37th Annual International Conference of Engineering in Medicine and Biology Society, pp. 4970-4973, IEEE Press, Milan, Italy (2015). doi: 10.1109/EMBC.2015.7319507. 